\title{
Gaseous Laser Targets and Optical Diagnostics for Studying Compressible Hydrodynamic Instabilities
}

\author{
J. Edwards, H. Robey, A. Mackinnon
}

June 29, 2001

U.S. Department of Energy

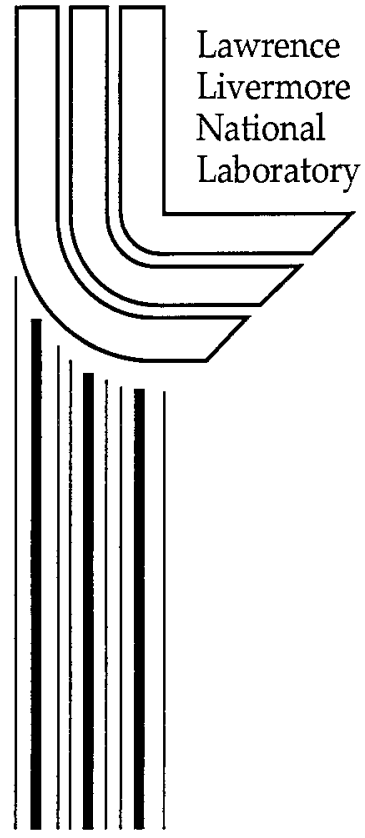




\section{DISCLAIMER}

This document was prepared as an account of work sponsored by an agency of the United States Government. Neither the United States Government nor the University of California nor any of their employees, makes any warranty, express or implied, or assumes any legal liability or responsibility for the accuracy, completeness, or usefulness of any information, apparatus, product, or process disclosed, or represents that its use would not infringe privately owned rights. Reference herein to any specific commercial product, process, or service by trade name, trademark, manufacturer, or otherwise, does not necessarily constitute or imply its endorsement, recommendation, or favoring by the United States Government or the University of California. The views and opinions of authors expressed herein do not necessarily state or reflect those of the United States Government or the University of California, and shall not be used for advertising or product endorsement purposes.

This work was performed under the auspices of the U.S. Department of Energy by the University of California, Lawrence Livermore National Laboratory under Contract No. W-7405-Eng-48.

This report has been reproduced directly from the best available copy.

Available to DOE and DOE contractors from the Office of Scientific and Technical Information

P.O. Box 62, Oak Ridge, TN 37831

Prices available from (423) 576-8401 http://apollo.osti.gov/bridge/

Available to the public from the National Technical Information Service

U.S. Department of Commerce 5285 Port Royal Rd., Springfield, VA 22161 http://www.ntis.gov/

\section{OR}

Lawrence Livermore National Laboratory Technical Information Department's Digital Library http://www.llnl.gov/tid/Library.html 


\title{
Gaseous laser targets and optical diagnostics for studying compressible hydrodynamic instabilities
}

\author{
John Edwards, Harry Robey, and Andrew Mackinnon \\ Lawrence Livermore National Laboratory, Livermore, CA 94550
}

\section{Objective:}

Explore the combination of optical diagnostics and gaseous targets to obtain important information about compressible turbulent flows that cannot be derived from traditional laser experiments for the purposes of $\mathrm{V} \& \mathrm{~V}$ of hydrodynamics models and understanding scaling.

First year objectives:

Develop and characterize blast wave - gas jet test bed

Perform single pulse shadowgraphy of blast wave interaction with turbulent gas jet as a function of blast wave Mach number

Explore double pulse shadowgraphy and image correlation for extracting velocity spectra in the shock-turbulent flow interaction

Explore the use/adaptation of advanced diagnositcs

\section{Introduction}

Compressible turbulent mix is a complex subject with many unresolved questions, and it will remain an active area of study for many years. There is as yet no firm consensus as to the role of initial conditions or compressibility on the evolution of a mixing region, and current state of the art simulations predict different values for the growth rate of mixing regions. To help resolve these issues compressible experiments are necessary. Another important question concerns what is typically referred to as the intensification of turbulence due to shock-turbulence interaction [eg see 1]. It is known that the passage of shocks through already turbulent flows increases the turbulent kinetic energy, but experiments only exist for incompressible experiments (Mach No., $M \sim 1)$. Experiments that measure what happens in the compressible regime $(M \gg>1)$ would be unique and ground-breaking.

Much work has been done on incompressible or low Mach number (M) flows in shock tubes and other devices such as the linear electric motor [see eg 2], while most work on compressible or high Mach number flows has been confined to energetic drivers such as lasers [see eg 3]. The former uses gases or liquids and powerful optical (and sometimes electro-mechanical) diagnostic techniques, and produce very high quality information, but at low Mach number. On the other hand, the information that can be obtained from current laser driven high Mach number hydrodynamics experiments, which use solid targets and foams, is limited by the need to use Xray diagnostics. These experiments do well at providing the shape of gross $2 \mathrm{D}$ structures (see fig. 1) which we model well, but are a long way from being able to reveal detailed information at the smaller spatial scales, or in 3D turbulent flows, where most of the modeling uncertainties exist. Remedying this is, and will continue to be, an ongoing research effort, but is unlikely to be easily accomplished in the near to medium term with X-ray diagnostics if at all.

One approach that is not being considered is to combine the compressibility offered by energetic laser drives with the gaseous targets and powerful diagnostic techniques developed in

\footnotetext{
${ }^{1}$ An exception is some form of 2-color radiography or spectroscopy to obtain the distribution of two materials in a mix region, averaged along the diagnostic line of sight. This was partially successful on Nova and should be realized soon on Omega.
} 
the low Mach number community. Although initial estimates are promising, research and development is required in order to establish how to adapt these techniques to high Mach number situations. There are also techniques such as optical shadowgraphy that have been routinely used in laser experiments and could be employed in compressible turbulent hydrodynamics experiments provided we use gaseous targets. We propose a small research effort to explore the use of techniques established in the laser arena such as optical interferometry (absolute electron density), and Schlieren photography (electron density gradient), to study compressible hydrodynamic instabilities in gaseous targets. We also propose to explore whether advanced techniques such as laser induced fluorescence (LIF) may be adapted to the plasma regime, thus providing detailed information, particularly about turbulent flows, that is not currently obtainable in plasmas using X-ray diagnostics. The setting will be simple blast waves, which can exhibit complex hydrodynamic instabilities while avoiding potentially costly target fabrication development. A high velocity gas jet would provide a turbulent flow for the blast wave to interact with. We propose to conduct the work in collaboration with Dr Todd Ditmire at the University of Texas at Austin, principally on the Janus laser, and Ditmire's short pulse laser, which is expected to be operational towards the beginning of FY02. His facility will be ideal for exploring diagnostic development. Dr Stephen Rose at AWE has expressed interest in collaboration and would provide computational support. He would also look into using the Helen laser at AWE, and developing a UK university contact, which may eventually open the possibility of shots on the Vulcan laser at RAL. We propose hiring a post doc to work full time on developing and exploring diagnostic techniques.

\section{First year research objective}

Work over the first year will focus on characterizing the blast wave and turbulent gas jet using established techniques. We will then measure the structure in the post shocked jet using shadowgraphy. By progressively increasing the separation between the gas jet and origin of the blast wave we will scan from Mach number $>10$ down to $\sim 1$ to overlap with existing data some of which has been obtained in shock tubes using shadowgraphy (see fig 2). We would also investigate double pulse shadowgraphy to attempt to correlate structures seen at the two different times thereby enabling us to extract velocity as a function of spatial scale, which is an important parameter for turbulence modelers. In parallel we will investigate advanced optical diagnostics with a view to implementing one in the following year. The diagnostics that will be investigated are laser induced fluorescence LIF[see eg 4, and fig 1], laser doppler velocimetry LDV[see eg. 5], and Rayleigh or coherent scattering which can provide absolute density information and is the most straightforward diagnostic. Of these, LDV may allow us to extract the turbulent kinetic energy spectrum and its evolution. This would be an important development for model developers. We show below that the conditions we can create on even the Janus laser might allow us to progress with these diagnostics.

\section{Experimental arrangement}

The experimental arrangement is very simple. The blast wave is formed by focussing a laser beam from Janus onto a thin foil of material, which sits in a background gas of for example nitrogen which fills the Janus target chamber. This type of experiment has been performed in the past [see eg 6]. The peak particle density that can be used depends on the intensity of laser light required at the thin foil. It turns out that it is not necessary to use very high intensities, but only enough to ensure that a reasonable fraction of the energy is absorbed since this is the important parameter for blast waves. Thus laser intensities $\sim 10^{12} \mathrm{Wcm}^{-2}$ should suffice. This has the added advantage of minimizing $X$-ray production, which might otherwise preheat the ambient gas and reduce the Mach number of the experiment. Under these condition, particle densities $10^{18}$ per cc are conservative ( $\sim 5 \%$ atmospheric pressure). A gas jet is positioned some distance from the foil and the blast wave is allowed to roll over the turbulent jet of gas. Optical shadowgraphy, Schlieren imaging and interferometry will be used to characterize the blast wave [see eg 7], the initial state of the turbulent jet, and the effect of the shock wave. By moving the gas jet further away from the 'explosion' which creates the blast wave we will be very easily 
able to scan in Mach number from $M>10$ to $M \sim 1$, thereby overlapping with experimental conditions that have already been accessed.

\section{Prospects for advanced diagnostics for $\mathrm{M} \sim 10$}

A potential obstacle for high Mach number laser experiments is their physical scale, which reduces the number of particles that can scatter a diagnostic laser beam to the detector. However, below we show that even for modest energy lasers such Janus, the physical scale is much larger than typical laser experiments and comparable to those of shock tubes in which these diagnostics have been shown to work.

The evolution of a simple (Sedov-Taylor) spherical blast wave is a classical problem and well understood [8]. The trajectory of the shock front is given by

$$
R=\alpha t^{2 / 5} ; \quad \alpha=\left[\frac{4}{\pi} \frac{(\gamma+1)^{2}(\gamma-1)}{3 \gamma-1}\right]^{1 / 5}\left(\frac{E}{\rho}\right)^{1 / 5}
$$

The radius at which the leading shock front attains a given velocity, $\mathrm{v}, \mathrm{can}$ therefore be written as

$$
R_{V}=\left(\frac{2}{5}\right)^{2 / 3}\left[A\left(\frac{E}{\rho}\right)\right]^{1 / 3} V^{-2 / 3} \approx 10^{4}\left(\frac{E_{\text {joule }}}{\left[n_{\text {percc }} / 10^{18}\right] A}\right)^{1 / 3} V_{c m / s}-2 / 3 \mathrm{~cm}
$$

where $\mathrm{n}$ is the particle density and $\mathrm{A}$ the molecular weight ( $\sim 30$ for air). The sound speed of air, which is mostly nitrogen, at room temperature is $\sim 3 \times 10^{4} \mathrm{~cm} / \mathrm{s}$, and a shock moving at Mach 10 would have a speed $\sim 3 \times 10^{5} \mathrm{~cm} / \mathrm{s}$. If we take $E \sim 100 \mathrm{~J}$, (which corresponds to a laser such as the current Janus), a background gas number density $\sim 10^{18}$ per $\mathrm{cc}$, we see from the above relation that the wave would have slowed to $\mathrm{Mach} \sim 10$ at a radius $\sim 3 \mathrm{~cm}$. Compared to typical laser targets this is macroscopic and not very different from typical shock tubes. This is significant because the number of particles that can scatter is also therefore not very different from shock tubes in which the powerful optical diagnostics we propose to investigate have been demonstrated. Because of the slow energy scaling, $E^{1 / 3}$, the physical size is changed little if we were to use the AWE Helen laser or the proposed Janus upgrade $(\sim 1 \mathrm{~kJ})$. On the other hand, an experiment transported to Omega would be $>10 \mathrm{~cm}$ radius, and $50 \mathrm{~cm}$ scales could be realized on the NIF.

It is also instructive to consider the temperature behind a $M \sim 10$ shock wave travelling through air initially at room temperature. For such a high Mach number the pressure ratio at the shock is approximately $2 \gamma \mathrm{M}^{2} /(\gamma+1) \sim \mathrm{M}^{2}$ for air with $\gamma \sim 1.4$. The compression through the shock is $\sim 6$ and the temperature increase is therefore a factor of $\sim 10-15$ corresponding to a temperature of less than $1 / 2 \mathrm{eV}$. This is low enough that optical pumping of transitions remains a possibility.

The above estimates show that adapting advanced diagnostics to high Mach number flows is worth investigating experimentally particularly considering the high potential payoff.

\section{Future prospects for gas targets}

Gaseous laser targets are attractive because they permit powerful optical diagnostics to be used. The lower density also allows the target to be very much bigger. These facts combined means that the range of scale that is resolvable is very much more than would be feasible using $X$-ray diagnostics and solid targets (in fact the actual spatial resolution of optical diagnostics is at least as good and typically better than achievable with X-rays). For turbulent flows this fact is very important and a major advantage of the proposed methods. On the other hand, gases do not 
lend themselves easily to complicated targets. Thus while we envisage more complicated experiments in future using gas cells on Omega and the NIF it is first necessary to demonstrate and develop the techniques in a simpler and more cost effective fashion.

\section{Potential Impact}

This work should have a significant impact on validation of hydrodynamics algorithms and models. It should provide the first measurements of high Mach number shock waves with flows that are already turbulent. It should also pave the way towards well-controlled experiments on the NIF to help resolve the role of compressibility and initial conditions on the development of turbulent mixing regions, and help us gain a necessary and sufficient understanding of the scaling of transient turbulent flows.

a)

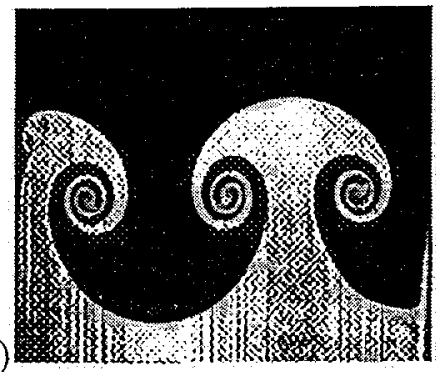

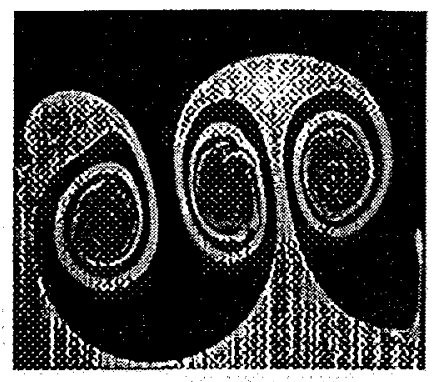

b)

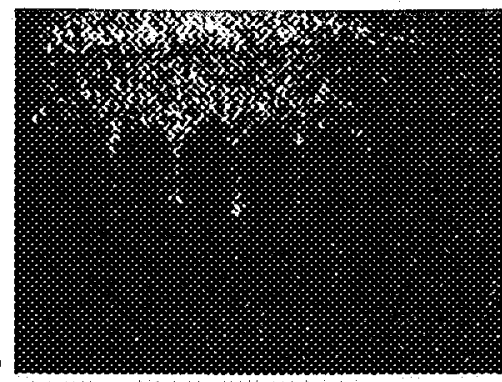

Fig 1 a) Example of high resolution LIF images from Jocob's M 1.2 shock tube experiments of two snapshots showing remarkable detail of development towards turbulence in the roll up of the mushroom cap. b) Example of near state-of-the-art high quality X-ray radiograph of Rayleigh-Taylor spikes in a M>10 Omega 'shock tube' experiment. The gross features are easily seen, but structure at smaller scales cannot be resolved.

Figure 2. Shadograph from Hesselink and Sturtevant, JFM 196, 513 (1988) showing intensification of turbulence due to the passage of a $\mathrm{M} \sim 1.1$ shock from left to right.

Post-shock

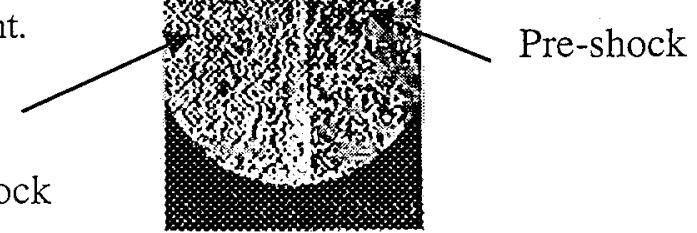

\section{References}

1) A Hokan and J Andreopoulos, Phys fluids A4(11) 2562, (1992).

2) G Dimonte, Phys. Plasmas 6, 2009 (1999).

3) J Edwards et al, Phys Plasmas 7, 2099, (2000).

4) F. Poggi et al, in Proceedings of the sixth workshop on the Physics of Compressible Turbulent Mixing Marseille France, (1997)

5) J W Jacobs and C E Niederhuas, ibid

6) J Grun et al, PRL 66, 2738, (1991)

7) J Edwards accepted for publication in PRL (2001)

8) L. I. Sedov, Similarity and Dimensional Methods in Mechanics, Academic Press 1997 (New York); G I Taylor, Proc. Royal Soc. A201, 159, (1950).

This work was performed under the auspices of the U.S. Department of Energy by the University of California, Lawrence Livermore National Laboratory under Contract No. W7405-Eng-48. 


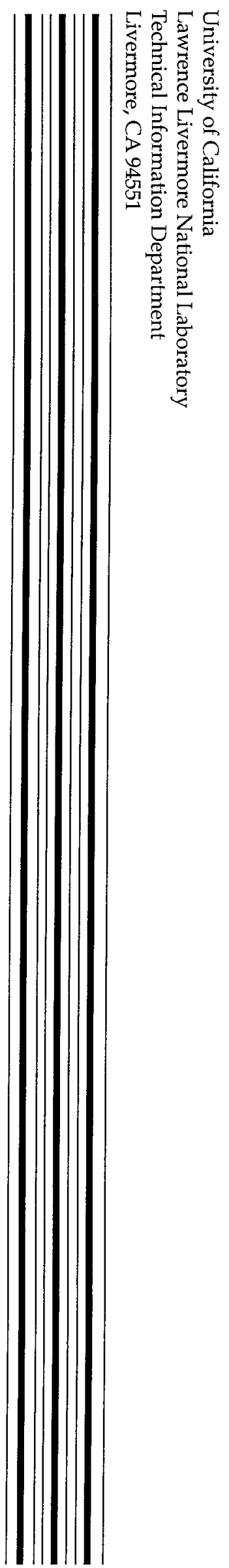

